\title{
SQUID Gradiometry for Magnetocardiography Using Different Noise Cancellation Techniques
}

\author{
M. Bick, K. Sternickel, G. Panaitov, A. Effern, Y. Zhang and H.-J. Krause
}

\begin{abstract}
Magnetocardiographic (MCG) measurements in unshielded environment require efficient noise cancellation techniques. We have applied two software gradiometry methods to analyze the time series of signal and reference data recorded outside magnetic shielding with high temperature superconducting quantum interference device (HTS SQUID) based gradiometers. One method uses adaptive frequency dependent gradiometer coefficients determined in the Fourier domain to subtract the reference from the signal data. The other method combines recently developed techniques for nonlinear projection with properties of the wavelet transform to extract noise in state space. The analyzed MCG data sets showed improved signal-to-noise ratios for both methods as compared to the data recorded with the electronic gradiometer. In this way, it is possible to increase the bandwidth from $130 \mathrm{~Hz}$ for our electronic gradiometer to $250 \mathrm{~Hz}$ without using any additional filtering.
\end{abstract}

Index Terms-Gradiometry, MCG, Noise cancellation

\section{INTRODUCTION}

$\mathrm{M}$ CG measurements are generally performed inside magnetically shielded rooms, e.g. [1], [2]. This is the best known method to suppress environmental noise mainly caused by power line disturbances. As these rooms are expensive and immobile, it is desirable to record MCGs outside shielding. Therefore, HTS SQUID gradiometers with additional filter techniques have been developed. Low-pass filters with cut-off frequencies of less than $130 \mathrm{~Hz}$ and additional notch filters are commonly employed [3]-[5]. Since notch filters destroy signal information, and standards for data-acquisition and analysis of high resolution ECG and MCG measurements recommend a bandwidth of $250 \mathrm{~Hz}$ without the use of notch filters [6], [7], improved noise cancellation techniques are required.

Hardware, electronic and software gradiometry as well as combinations thereof [2], [4], [5], are the fundamental basis for further improvements. Standard software gradiometry uses reference sensors with amplitude $b_{i}$ to subtract environmental noise from signal sensors with amplitude $a$ located close to

Manuscript received September 18, 2000.

Marcel Bick, Grigory Panaitov, Yi Zhang and Hans-Joachim Krause are with the Institut fur Schicht- und lonentechnik, Forschungszentrum Jülich, 52425 Jülich, Germany (phone +492461613522, email m.bick@fzjuelich.de).

Karsten Sternickel and Arndt Effern are with the Institut fïr Strahlen- und Kernphysik, Universtăt Bonn, 53115 Bonn, Germany (phone +49228733008, email stern@iskp.uni-bonn.de) the signal source [8]. Constant coefficients $k_{i}$ are determined dependent on the local noise environment before the measurement [9], so that a noise reduced output $s=a-\sum k_{i} \cdot b_{i}$ is generated. Because environmental noise varies quickly in phase and amplitude during the measurement, it is advantageous to determine the coefficients adaptively. Considering this, we employed two adaptive software noise cancellation techniques each requiring a signal sensor located close to the human heart and at least one reference sensor mainly measuring environmental noise. In the following section, both methods will briefly be described. We performed MCG measurements in a magnetically unshielded environment using an electronic HTS SQUID 2nd order gradiometer (SOG) and applied both software techniques. We will show that it is possible to increase the signals' bandwidth from $130 \mathrm{~Hz}$ to $250 \mathrm{~Hz}$ without using any notch filters.

\section{APPLIED SOFTWARE NOISE CANCELLATION TECHNIQUES}

\section{A. Frequency Dependent Gradiometry (FDG)}

In contrast to standard software and electronic gradiometry, FDG uses frequency dependent coefficients $k_{i}(f)$ to subtract the reference from the signal data [10]. These coefficients are adaptively determined during the measurement. To calculate $k_{i}(f)=\left\{k_{S i}(f), k_{N i}(f)\right\}$, the time series of signal and reference sensors are Fourier transformed using overlapping Hanning windows. Here, $k_{s i}(f)$ denotes the ratio between the amplitudes of the source signal in measuring sensor and reference sensor $i$ and $k_{N i}(f)$ denotes the ratio of the external noise amplitudes. The complex amplitude of the source signal $a^{s}(f)$ can be computed for a given time window by:

$$
a^{s}(f)=\frac{k_{S i}(f)}{k_{S i}(f)-k_{N i}(f)}\left[a(f)-k_{N i}(f) b_{i}(f)\right] .
$$

All values are complex numbers. The measured values $a(f)$ of the signal sensor are replaced by the values $a^{s}(f)$ only at frequencies where noise has been detected. Averaging complex amplitude products of $a(f)$ and $b_{i}(f)$ over several time windows, $k_{S i}(f)$ and $k_{N i}(f)$ can be determined by $k_{S i}=$ $\left(a^{S} \cdot b^{S}{ }_{i}\right) /\left(b_{i}^{S_{i}} \cdot b^{S}{ }_{i}^{*}\right)$ and $k_{N i}=\left(a^{E N} \cdot b^{E N}{ }_{i}\right) /\left(b^{E N}{ }_{i} \cdot b^{E N}{ }_{i}^{*}\right)$ where $a^{E N}$ and $b^{E N}{ }_{i}$ denote the external noise in the sensors and $*$ denotes the complex conjugate [10]. The number of averages determines the standard deviation $\sigma_{k}$ of the calculated coefficients $k_{i}(f) . \sigma_{k}$ will decrease when a large time interval $\tau_{A}$ for recalculation of $k_{i}(f)$ is chosen. This will reduce the rate of adaptation to changing noise sources. Therefore, the tuning parameters $\tau_{A}$ and $\sigma_{k}$ have to be optimized with respect to external disturbances. At low frequencies $\mathrm{f}<\mathrm{f}_{\mathrm{L}}$ where 
external $1 / \mathrm{f}$ noise dominates periodic noise, $k_{i}(f)$ is set to a constant value because the coefficients can be calculated with sufficient accuracy for periodic disturbances only.

\section{B. Nonlinear Denoising in State Space (NLD)}

NLD operates on the reconstructed state space of the time series which represents the dynamical properties of the observed system. An 'at least approximately' deterministic system leads to densely lying trajectories and is constrained to a subspace whereas a stochastic process causes a random distribution within the entire state space (see Fig. 1a). Superimposing white noise leads to distortions of the primary densely lying MCG trajectories in state space (see Fig. 1b). We correct this by projecting these trajectories towards the direction of the maximal variance effectuated by the determinism of the MCG (Fig. 1c). Deterministic noise mainly caused by powerline disturbances fills additional subspaces which have to be separated from the MCG manifold. By recording the deterministic noise in an additional reference sensor and - due to the time varying character of this noise - using the adaptive basis functions of the wavelet transform to find its according subspace, we identify this signature in the time series of the source sensor and obtain an improved noise reduction. Further details on the method can be found in [11].
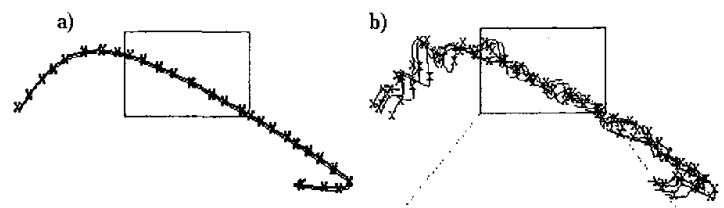

c)

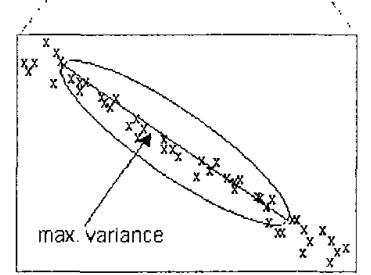

Fig. 1. a) Densely lying MCG trajectories in a 2 dimensional state space. In b) superimposed white noise expands the densely lying trajectories. The magnification of $b$ ) is shown in c). Points in state space are corrected by projecting them towards the direction of the maximal variance caused by the MCG determinism.

\section{EXPERIMENTAL}

To be able to compare the above described software techniques to electronic gradiometry with constant coefficients, we performed MCG measurements outside magnetic shielding in our laboratory environment. An SOG with baseline distances of $7.5 \mathrm{~cm}$ was employed [5]. The system consisted of 3 axially arranged HTS-rf-SQUIDs (Fig. 2) with coplanar resonators and a washer area of $8 \times 8 \mathrm{~mm}^{2}$. The system's typical magnetic field noise was $150 \mathrm{fT} / \sqrt{\mathrm{Hz}}$ (Fig. 3c). Common mode rejection for the two 1st order gradiometers of the system (FOG A and B in Fig. 2) was electronically balanced to better than $10^{4}$ for homogeneous fields. The background noise in our laboratory and the magnetic field noise of FOG and SOG are shown in
Fig. 3. For MCG measurements with the electronic SOG, a bandpass filter with a passband of $0.05-130 \mathrm{~Hz}$ and notch filters at 100 and $150 \mathrm{~Hz}$ were applied (Fig. 2a). For comparison with the software techniques a bandwidth of $250 \mathrm{~Hz}$ without notch filters was also used.

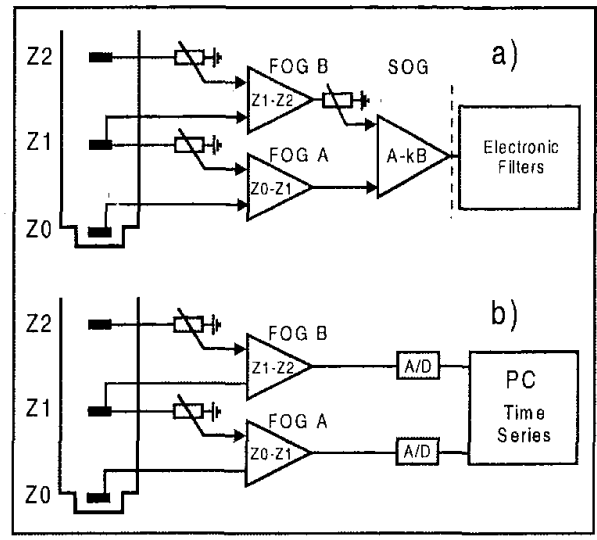

Fig. 2. Measurement setup outside magnetic shielding, a) electronically built SOG including hardware filters, b) application of software cancellation techniques.

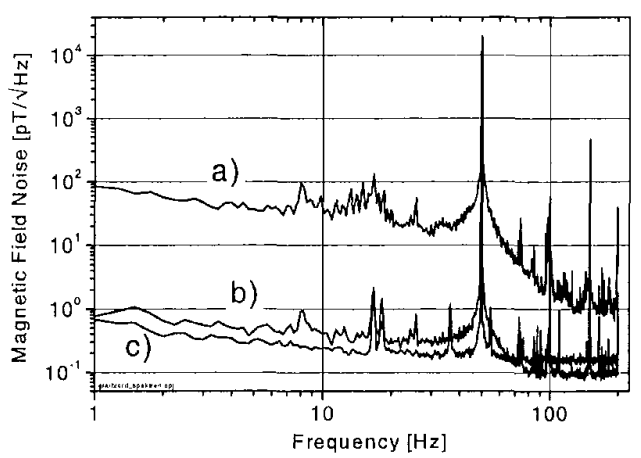

Fig. 3. Typical magnetic field noise outside shielding of a) magnetometer, b) FOG and c) SOG.

To apply the software noise cancellation methods we used FOG $B$ as a reference (Fig. 2b). The time series of reference (FOG B) and signal sensor (FOG A) were acquired simultaneously with a PC. A bandwidth of $250 \mathrm{~Hz}$ and a sample rate of $1 \mathrm{kHz}$ were used. For comparison, the time series of the electronically built gradiometers were recorded consecutively. Applying FDG and NLD, the time series of the signal sensor were corrected according to the denoising procedures described in section II. To solve equation (1), the following assumptions had to be made because only one reference sensor was used: $k_{S i}(f)$ is time independent and the ratio of the sensors' intrinsic noise amplitudes is constant. In our laboratory environment, the best signal-to-noise ratio (SNR) for FDG was achieved with $\tau_{\mathrm{A}}=15 \mathrm{sec}, \sigma_{\mathrm{k}}=5 \%$ of $\mathrm{k}_{\mathrm{i}}(\mathrm{f})$ and $\mathrm{f}_{\mathrm{L}}=5 \mathrm{~Hz}$. For NLD, an embedding dimension of $\mathrm{m}=512$, a time delay of $\tau=1$ data point, the number of nearest neighbors of $\mathrm{k}=20$, the coiflet 2 mother wavelet, and thresholding parameters of $\lambda_{R}=0.5$ and $\lambda_{S}=0.5$ were used. 


\section{RESULTS}

In Fig. 4, a comparison of real time MCG signals recorded in laboratory environment is presented. Here, we regard real time as non-averaged signals that may have a time delay due to signal processing. FDG (Fig. 4c) shows a significant improvement in SNR compared to the electronic SOG with a bandwidth of $250 \mathrm{~Hz}$ (Fig. 4a). A similar result can be obtained for the SOG only when a reduced bandwidth of $130 \mathrm{~Hz}$ and notch filters at 100 and $150 \mathrm{~Hz}$ are used (Fig. 4b). NLD with a bandwidth of $250 \mathrm{~Hz}$ shows an even more significant improvement (Fig. 4d).

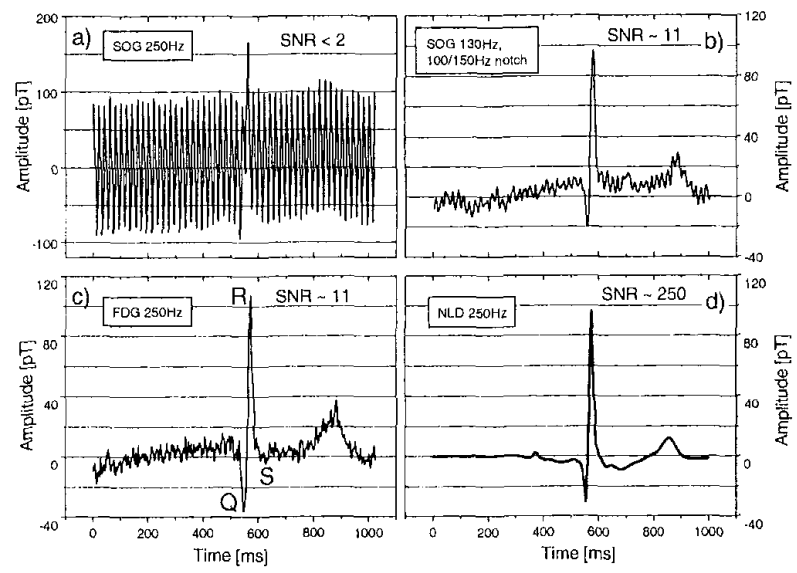

Fig. 4. Real time MCG signals recorded outside shielding: a) electronic SOG, $250 \mathrm{~Hz}$ bandwidth, b) electronic SOG with $130 \mathrm{~Hz}$ bandwidth and notch filters, c) FDG, $250 \mathrm{~Hz}$ bandwidth and d) NLD, $250 \mathrm{~Hz}$ bandwidth. The signals were recorded consecutively.

To investigate the preservation of the MCG signal, averaging of the still noisy FDG and SOG data is necessary. In Fig. 5, 100 times averaged FDG and SOG signals, the real time MCG signal obtained by NLD, and the corresponding Fourier spectra are compared. The enlarged QRS amplitude in Fig. $5 \mathrm{a}$ indicates a possible improved preservation of the heart beat for FDG. We can also note a considerable reduction of power line disturbances in the corresponding Fourier spectra (Fig. 5b). The real time NL.D data show a similar SNR as the 100 times averaged SOG data with a bandwidth of $130 \mathrm{~Hz}$ (Fig. 5c). The corresponding Fourier spectrum demonstrates a signal preservation at 100 and $150 \mathrm{~Hz}$ in contrast to the SOG (Fig. 5d). However, in Figs.5a and $c$, different shapes of the heart beat for NLD data as compared to FDG and SOG data can be observed. This deviation will be discussed in more detail in section $\mathrm{V}$.

\section{V.DISCUSSION}

A summarizing comparison of the three investigated noise compensation techniques is displayed in Table I. A better SNR for the software techniques has already been stated above. Some further advantages of FDG and NLD over electronic gradiometry need to be discussed in more detail. For FDG, a lower intrinsic noise level is achievable because the noise of the reference sensor is only added at frequencies
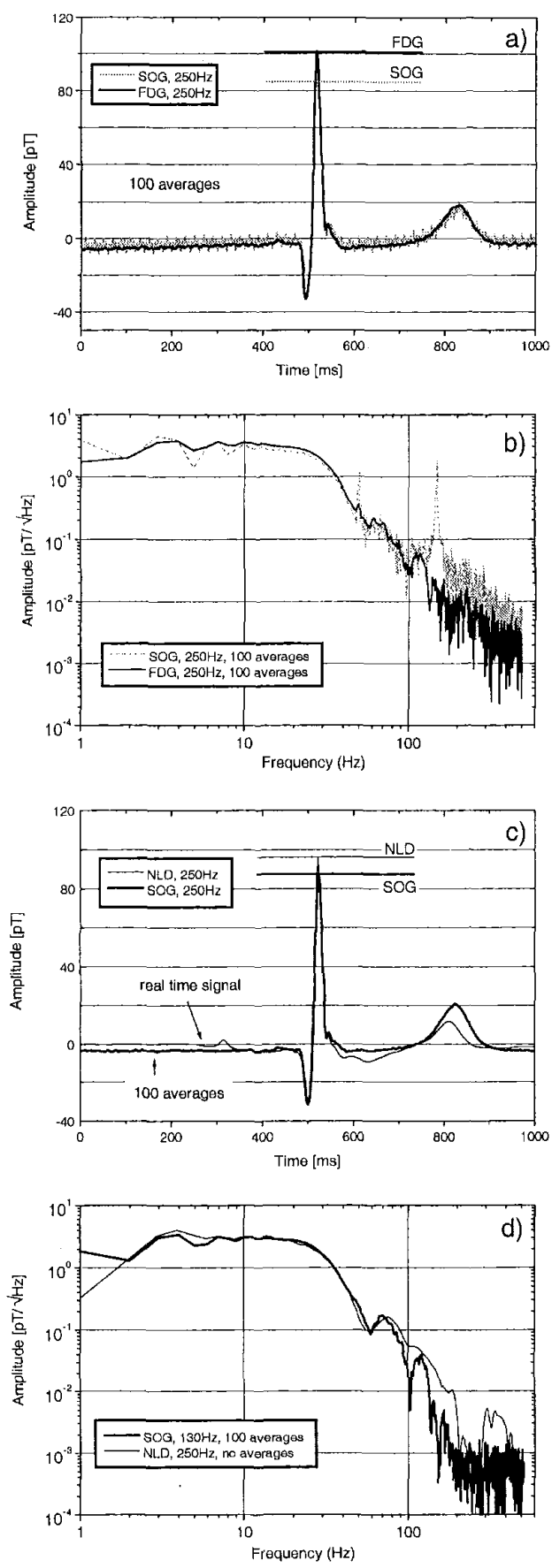

Fig. 5. MCG signals and corresponding Fourier spectra recorded outside shielding: a) comparison of averaged MCG signals using electronic SOG (thin line) and FDG (bolded line) with $250 \mathrm{~Hz}$ bandwidth without additional filters; $c$ ) comparison of real time MCG signal using NLD with $250 \mathrm{~Hz}$ bandwidth (thin line) and averaged MCG signal using electronic SOG with $130 \mathrm{~Hz}$ bandwidth and notch filters at 100 and $150 \mathrm{~Hz}$ (bolded line); b) and d) show the corresponding Fourier spectra.

where external disturbances have been detected (Fig. 5b). This can lead to a maximum noise reduction of $\sqrt{2}$ if reference and signal sensor have the same intrinsic noise. 
However, due to the need to average in the Fourier domain, the adaptation to temporal noise fluctuations is limited for FDG. Depending on the noise environment, $\sigma_{k}$ and $\tau_{A}$ have to be determined in a way that the SNR is maximized. A typical value of $\tau_{A} \sim 15-20 \mathrm{sec}$ for our laboratory has been found. NLD using reference data shows a significant improvement of the SNR by reducing the ambient noise and canceling the white noise by averaging in state space. By means of a wavelet transform, the adaptation to changing noise sources is improved: analyzing an MCG time series of 5 seconds a SNR of $\sim 250$ has been achieved for a non averaged MCG outside magnetic shielding (Fig. 4d).

TABLE I

Comparison of Presented Noise Cancellation Techniques

\begin{tabular}{lccccc}
\hline \hline Method & $\begin{array}{c}\text { Band- } \\
\text { width }\end{array}$ & $\begin{array}{c}\text { SNR** } \\
\text { for } \\
\text { MCG }\end{array}$ & $\begin{array}{c}\text { Online } \\
\text { Noise } \\
\text { Adaptation }\end{array}$ & $\begin{array}{c}\text { Tuning } \\
\text { Para- } \\
\text { meters }\end{array}$ & Processing \\
\hline SOG & $250 \mathrm{~Hz}$ & $<2$ & No & 3 & online \\
SOG & $130 \mathrm{~Hz}^{*}$ & $\sim 11$ & No & 3 & online \\
FDG & $250 \mathrm{~Hz}$ & $\sim 11$ & Yes & 3 & $\begin{array}{c}\text { time delay } \\
\sim 1 \text { min. } \\
\text { time delay } \\
\text { NLD }\end{array}$ \\
& $250 \mathrm{~Hz}$ & $\sim 250$ & Yes & 6 & 5 min. \\
\hline \hline
\end{tabular}

*notch filters @100 and $150 \mathrm{~Hz}$; **eal time signals, SNR = amplitude of heart signal / peak-to-peak noise

To investigate a possible signal distortion in applying FDG and NLD, we tested the two noise cancellation techniques inside a magnetically shielded room using an electronic 1 st order HTS SQUID gradiometer with a magnetic field noise of $35 \mathrm{fT} / \sqrt{\mathrm{Hz}}$ described in [12]. Inside magnetic shielding, MCG recordings can be performed with $250 \mathrm{~Hz}$ bandwidth without the use of notch filters. This guarantees a minimum of distortion and therefore the signal can be taken as a reference. For both software methods, an enlarged amplitude of the QRS complex and no distortion of the heart signals could be observed. These results confirm the better signal preservation in case of FDG outside shielding (Fig. 5a). For NLD, investigations on synthetically generated noisy ECG data showed sufficient signal preservation as well [11]. However, in the presence of enlarged magnetic disturbances, the determination of the 6 tuning parameters becomes much more difficult. It could be shown that the shape of the signal depends on the choice of the parameters. Therefore, they have to be determined very carefully to avoid a possible signal distortion.

\section{CONCLUSION AND OUTLOOK}

In summary we have presented two adaptive software gradiometry techniques and demonstrated their advantages over conventional electronically built gradiometers for MCG outside magnetically shielded rooms in moderate magnetic environment. The MCG time series of reference and signal sensors were analyzed by using frequency dependent gradiometry and nonlinear denoising, respectively. Both methods showed substantial reduction of external and intrinsic noise. We were able to extend the bandwidth of an electronic SOG from $130 \mathrm{~Hz}$ to $250 \mathrm{~Hz}$ without using notch filters, meeting the recommended standard for clinical MCG diagnostics. FDG shows a better preservation of the heart signal as compared to electronic gradiometry. NLD leads to a significant improvement of the SNR, thus allowing analysis of real time MCG signals outside magnetic shielding. However, we found a slight distortion of the MCG signal after applying NLD which is subject to further investigation. Future work will include a faster online calculation and an optimized determination of the free parameters of the methods to a given noise environment.

\section{ACKNOWLEDGMENT}

The authors would like to thank P. David for invaluable extensive discussions, E. Zimmermann and M. Schiek for assistance in data analysis, and A.I. Braginski for critically reading of the manuscript.

\section{REFERENCES}

[1] H.P.Müller, P.Gödde. K.Czerski, M.Oeff, R.Agrawal, P.Endt, W.Kruse, U.Steinhoff and L.Trahms, "Magnetocardiographic analysis of the twodimensional distribution of intra-QRS fractionated activation", Phys.Med.Biol. vol. 44, pp. 105-120, 1999.

[2] W.Andrae, H.Nowak (eds.), "Magnetism in medicine: a handbook", pp. 136-189, Wiley-VCH, Berlin 1998.

[3] J.Borgmann, P.David, G.Ockenfuss, R.Otto, J.Schubert, W.Zander, and A.I.Braginski, "Electronic high-temperature radio frequency superconducting quantum interference device gradiometers for unshielded environment", Rev.Sci.Instrum. vol. 68, pp. 2730-2734, 1997.

[4] K.A.Kouznetsov, J.Borgmann, and J.Clarke, "High-Tc second-order gradiometer for magnetocardiography in an unshielded environment", Appl.Phys.Lett. vol. 75 (13), pp. 1979-1982, 1999.

[5] Y. Zhang, G.Panaitov, S.G.Wang, N.Wolters, R.Otto, J.Schubert, W.Zander, H.-J.Krause, H.Soltner, H.Bousack, and A.I.Braginski, "Second-order, high-temperature superconducting gradiometer for magnetocardiography in unshielded environment", Appl.Phys.Lett. vol. 76 (7), pp. 906-908, 2000.

[6] G.Breithardt, M.E.Cain, N.Ei-Sherif, N.C.Flowers, V.Hombach, M.Janse, M.B.Simson, and G.Steinbeck, "Standards for analysis of ventricular late potentials using high-resolution or signal-averaged electrocardiography", Circulation vol. 83 (4), pp. 1481-1488, 1991.

[7] G.Stroink, M.J.R.Lamothe, and M.J.Gardener, "Magnetocardiographic and electrocardiographic mapping studies" in: Weinstock, H. (ed.), "SQUID Sensors: Fundamentals, Fabrication and Applications", pp. 413-444, Dordrecht NL, Kluwer Academic Press, 1996.

[8] J.Vrba, "SQUID gradiometers in real environments", in: Weinstock, H. (ed.), "SQUID sensors: Fundamentals, fabrication and applications", pp. 117-178, Dordrecht NL, Kluwer Academic Press, 1996.

[9] S.E.Robinson, "Environmental noise cancellation for biomagnetic measurements", in: S.J. Williamson et al. (eds.), "Advances in biomagnetism", pp. 721-724, Plenum Press, New York, 1989.

[10]F.Rüders, M.Bick, H.Soltner, D.Selbig, and H.Bousack, "Frequency dependent gradiometry: A new noninvasive method of improved noise cancellation applied to magnetocardiography", Biomedizinische Technik vol. 42, suppl. 1, pp.231-234, 1997.

[11] K.Sternickel, A.Effern, K.Lehnertz, Th.Schreiber, and P.David, "Non linear denoising using reference data", Phys.Rev.E. vol. 63 (2) 2001, in press.

[12] X.H.Zeng, H.Soltner, D.Selbig, M.Bode, M.Bick, F.Rüders, J.Schubert, W.Zander, M.Banzet, Y.Zhang, H.Bousack, and A.I.Braginski, "A hightemperature if SQUID system for magnetocardiography", Meas.Sci.Technol. vol. 9, pp. 1600-1608, 1998. 